\title{
MicroRNA-122 regulation of the morphology and cytoarchitecture of hepatoma carcinoma cells
}

\author{
JI-CHUN JIN, XIAN ZHANG, XING-LIN JIN, CHANG-SHI QIAN, HAO JIANG and YANG RUAN
}

Department of Hepatopancreatobiliary Surgery, Affiliated Hospital of Yanbian University, Yanji, Jilin 133000, P.R. China

Received June 4, 2013; Accepted January 16, 2014

DOI: $10.3892 / \mathrm{mmr} .2014 .1930$

\begin{abstract}
MicroRNAs (miRNAs) are a large family of post-transcriptional regulators of gene expression that control a number of developmental and cellular processes in eukaryotic organisms and are 23 nucleotides in length. miRNA-122 is an abundant liver-specific miRNA, implicated in fatty acid and cholesterol metabolism, as well as in hepatitis $\mathrm{C}$ viral replication and is frequently suppressed in primary hepatocellular carcinomas. In the current study, the Hep3B cell line with stable overexpression of miR-122 was successfully established through gene transfection methods and drug screening. miR-122 was observed to alter cell morphology in vitro by stable overexpression in Hep3B cells. This alteration was viewed by light microscopy and transmission electron microscopy. These alterations included increases in the cell volume, the appearance of lipid granules and vacuoles, thickening of nuclear membrane, swelling of the mitochondria, cytoplasm vacuolization and a more prominent nucleolus. Furthermore, the study provided novel evidence that miR-122 function was dependent upon its expression level. In addition, it was observed to negatively regulate mitochondria.
\end{abstract}

\section{Introduction}

Mature microRNAs (miRNAs) are single-stranded RNA molecules, between 20- and 23-nucleotides in length, that control gene expression in cellular processes. These molecules typically decrease the stability of mRNAs, including those of genes that mediate processes in tumorigenesis, including cell cycle regulation, differentiation, apoptosis, invasion and stress responses (1). miRNAs were initially linked to tumorigenesis due to their proximity to chromosomal breakpoints and their unregulated expression levels in a number of malignancies. Given their integral role in development, miRNAs were

Correspondence to: Professor Xing-Lin Jin, Department of Hepatopancreatobiliary Surgery, Affiliated Hospital of Yanbian University, 1327 Juzi Street, Yanji, Jilin 133000, P.R. China

E-mail: xljin@ybu.edu.cn

Key words: microRNA-122, hepatocellular carcinomas, Hep3B, cell morphology observed as being significant in tumorigenesis. miRNA targeting is achieved through specific base-pairing interactions between the 'seed' region of the miRNA and sites within coding and untranslated regions of mRNAs. Target sites in the 3' untranslated region (UTR) lead to more effective mRNA destabilization.

As the most abundant liver-specific miRNA, miR-122, was identified as constituting $70 \%$ of total hepatic microRNA, while cloning small RNAs from different tissues of the body $(2,3)$. Its expression was activated gradually with the growth of embryonic development. miR-122 is implicated in fatty acid and cholesterol metabolism, and depletion of miR-122 by an antisense oligopeptide lowers the cholesterol level in plasma (4). miR-122 is also required by the hepatitis $\mathrm{C}$ virus and is frequently suppressed in primary hepatocellular carcinomas (HCCs) (5).

To determine the physiological roles of miR-122 and the biotransformation of hepatoma carcinoma cells highly expressing miR-122, the Hep3B cell line, which stably overexpressed miR-122, was generated by gene transfection methods and drug screening.

\section{Materials and methods}

Cell cultures. Hep3B was obtained from the Chinese Academy of Sciences Cell Bank (Shanghai, China) and the cell line was maintained in Dulbecco's modified Eagle's medium supplemented with $10 \%$ fetal bovine serum. A humidified incubator was set at $37^{\circ} \mathrm{C}, 5 \% \mathrm{CO}_{2}$.

Plasmid construction. The oligonucleotide chains Pre-miR-122-1 and Pre-miR-122-2, which contained SmaI and EcoRI endonucleases, were designed by precursor sequences of has-miR-122 from miRBASE (http://www.mirbase. org/cgi-bin/mirna_entry.pl?acc=MI0000442) and were as follows: PreMIR-122-1, 5'-TCCCCCGGGGGACCGGGA TCCGCCTTAGCAGAGCTGTGGAGTGTGACAATGGTG TTTGTGTCTAAACTATCAAACG-3'; and PreMIR-122-2, 5'-TCCCCCGGGGGACGGAATTCAAAAAAGGCCTAGCAG TAGCTATTTAGTGTGATAATGGCGTTTGATAGTTTAG CA-3' (restriction sites indicated by underlining). The 3'UTR of miR-122-Luc, containing the miR-122 response element (miR-122-Luc-1, 5'-CTAGAACAAA CACCATTGTCACACTCCAT-3'; miR-122-Luc-2, 5'-CTA GATGGAGTGTGACAATGGTGTTTGTT-3'), was cloned 
Table I. Quantitative polymerase chane reaction primers.

\begin{tabular}{lll}
\hline Gene & \multicolumn{1}{c}{ Primer } & \multicolumn{1}{c}{ Sequence } \\
\hline miR-122 & Reverse transcription & 5'-CTCAACTGGTGTCGTGGAGTCGGCAATTCAGTTGAGCAAACAC-3' \\
& Forward & 5'-ACACTCCAGCTGGGTGGAGTGTGACAATGGTGTTTG-3' \\
& Reverse & 5'-CTCAACTGGTGTCGTGGA-3' \\
U6 & Forward & 5'-CTCGCTTCGGCAGCACA-3' \\
& Reverse & 5'-AACGCTTCACGAATTTGCGT-3' \\
\hline
\end{tabular}

U6 was used as a reference gene.

A

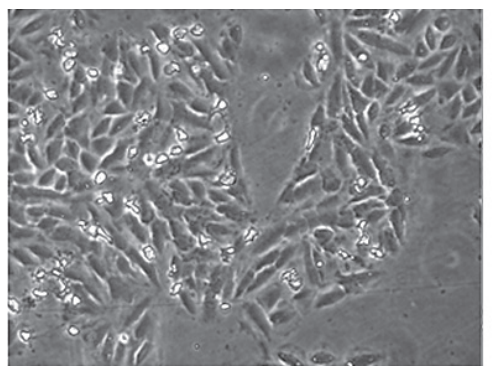

B

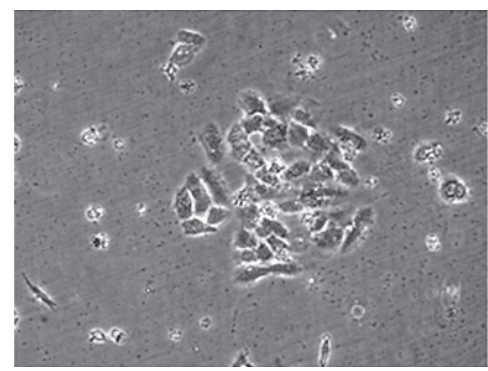

C

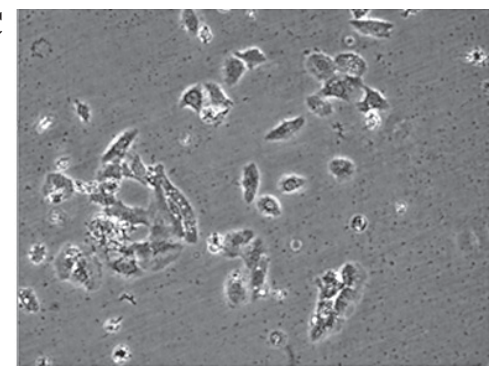

Figure 1. There was no significant change in the cellular morphology following transfection (48 h). (A) Hep3B, (B) Hep3B-122 and (C) Hep3B-Q. Magnification, x40.

into the pGL-control vector plasmid (Promega Corporation, Madison, WI, USA), which contained the XbaI endonuclease. The double-strand was cloned into pGL-control vector to acquire the recombinant plasmid pGL3-miR122-Luc.

Stable transfection of miR-122. pSIREN-miR-122 was transfected into Hep3B cells using Effectene ${ }^{\circledR}$ Transfection Reagent (Qiagen, Hilden, Germany) according to the manufacturer's instructions. After $24 \mathrm{~h}$, the fresh culture medium was replaced with $2 \mu \mathrm{g} / \mathrm{ml}$ puromycin to obtain the positive monoclonal cells which expressed stably (Hep3B-122 cells). Hep3B-pSIREN-RetroQ cells were generated by transfecting Hep3B cells with RNAi-Ready pSIREN-RetroQ vector as the plasmid control group (Hep3B-Q).

Dual-luciferase reporter assay. The 3'UTR fragments of the candidate target genes were subcloned into the $\mathrm{XbaI}$ site downstream of the luciferase gene in the pGL3-control vector (Promega Corporation). Under the control of the SV40 promoter and enhancer, pGL3-control vector expresses marked luciferase activity in numerous types of mammalian cells. To verify pSIREN-miR-122's activity in cells, pGL3-miR122-Luc, pSIREN-miR-122 and pRL-SV40 were transfected in Hep3B at the same time using a Dual-Luciferase ${ }^{\circledR}$ Reporter assay System kit (Promega Corporation) according to the manufacturer's instructions. The luciferase activity of Firefly and Renilla was obtained from pGL3-miR122-Luc and pRL-SV40 by Infinite 200 Pro multimode plate readers (Tecan Systems Inc., San Jose, CA, USA).

$q P C R$. RNA was harvested using TRIzol (Invitrogen, Hong Kong, China) according to the manufacturer's instructions. Quantification of total RNA was performed using a NanoDrop ND-1000 spectrophotometer (NanoDrop, Thermo Scientific,
Wilmington, DE, USA). To analyze miRNA expression, PCR master mix (2X; Superarray, Valencia, CA, USA) was used to quantify levels of mature miRNAs according to the manufacturer's instructions. The PCR reaction was as follows: enzyme activation for $10 \mathrm{~min}$ at $95^{\circ} \mathrm{C}$ followed by 40 cycles of $95^{\circ} \mathrm{C}$ for $15 \mathrm{sec}$ and $60^{\circ} \mathrm{C}$ for $60 \mathrm{sec}$. Primers are shown in Table I. Data was analyzed using the $2^{-\Delta \Delta \mathrm{CT}}$ method.

\section{Results}

Cell reproduction rate was decreased when visualized following $48 \mathrm{~h}$ transfection; however cellular morphology did not exhibit significant differences (Fig. 1). Ten hours later ( $\sim 60 \mathrm{~h}$ following transfection), there was a significant morphological change observed at high magnification (Fig. 2). A significant boundary between the cytoplasm and nucleus was observed with the increase in cell volume. In addition, the nucleolus and nuclear matrix/skeleton were enlarged and easy to recognize. The nucleolus was more easily distinguishable compared with in the control group. Notably, the cavitating structures of varying sizes appeared throughout the cytoplasm and increased in size with increased proximity to the nucleus.

To utilise the puromycin resistance the RNAi-Ready pSIREN-RetroQ vector possesses, the Hep3B cell lines were obtained with stable high expression of miR-122 by high intensity-drug screening. To verify the content and activity expressive of miR-122 in cells, a Dual-Luciferase ${ }^{\circledR}$ Reporter assay and quantitative PCR were performed (Fig. 3). Compared with the control group, the pSIREN-miR-122 plasmid was capable of expressing the mature series of miR-122 abundantly and miR-122 efficiently performed biological functions that inhibited the expression of the target gene. 

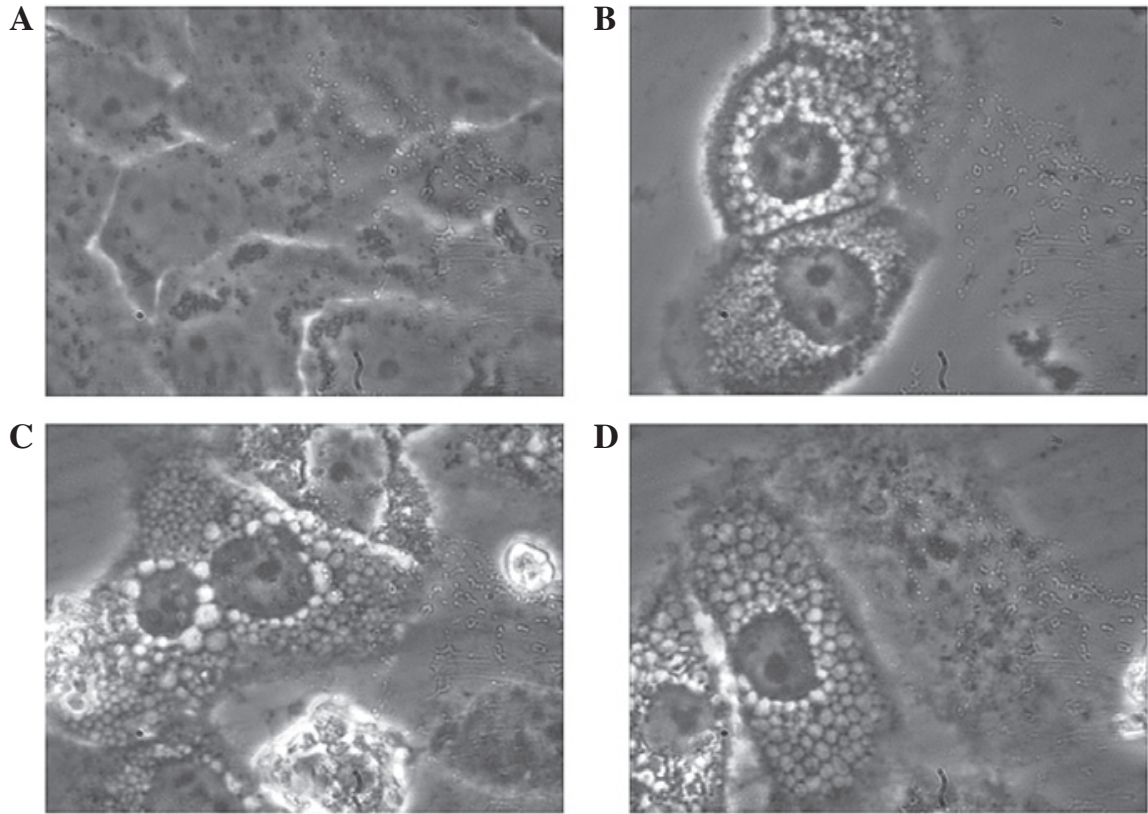

Figure 2. Following transfection $(60 \mathrm{~h})$, (A) the control group exhibited no significant natural alteration, however a significant boundary between the cytoplasm and nucleus was observed in Hep3B-122 at $72 \mathrm{~h}$ after transfection (B, C and D). (B) Enlargement of the nucleolus and increase in nuclear matrix/skeleton. (C) Notably the cavitating structures of varying sizes appeared throughout the cytoplasm and structures in closer proximity to the nucleus appeared larger. (D) It was possible to determine which cells express the miR-122 gene. Magnification, $\mathrm{x} 400$.

A

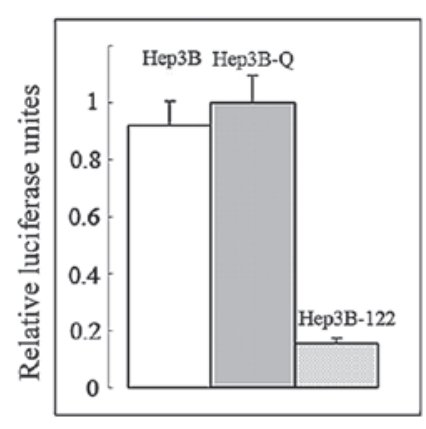

C

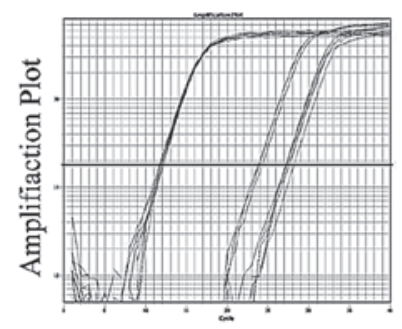

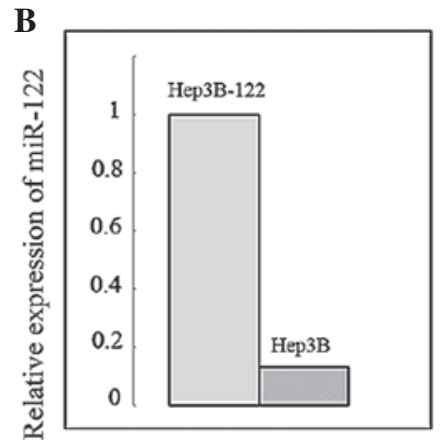

D

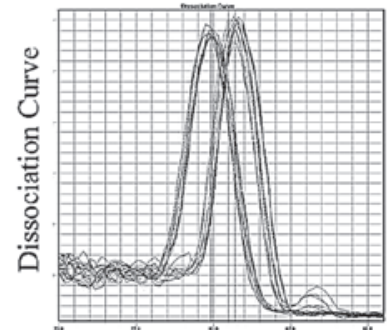

Figure 3. To verify the content and activity of miR-122 expression in Hep3B-122 cells following drug screening, (A and B) Dual-Luciferase Reporter assay and (C and D) quantitative polymerase chain reaction (qPCR) were performed. The activity of miRNA-122 was sixfold higher in Hep3B-122 cells compared with Hep3B cells and expression was sevenfold higher.

In the stage of stable high expression of miR-122 following drug screening and miR-122 detection, Hep3B-122 cells showed further characteristic features (Fig. 4). Simultaneous to the increase in volume, cells swelled and expanded and the edges became smooth and bright. In addition, similar to a lipid granule, a transparent droplet gathered at the edge or two poles of the cells and a vacuole appeared in the transfection phase $(\sim 60 \mathrm{~h}$ after transfection) and gradually integrated into larger vacuoles in the center.
To further identify this transformation, the Hep3B-122 group and the control group were scanned by transmission electron microscopy and compared with the control groups (Fig. 5). The nucleus was observed to be enlarged, the nucleus was located at the edge of the nucleus and the quantity of heterochromatin decreased. In addition to the swelling of mitochondria, the double membrane structure was not clear as the mitochondria exhibited cavities. Furthermore, the cytoplasm was vacuolated and the microvilli on the cell membrane were damaged. 


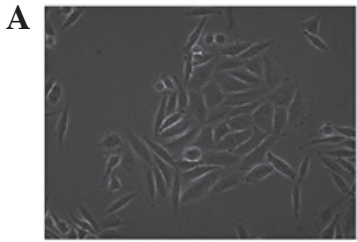

F

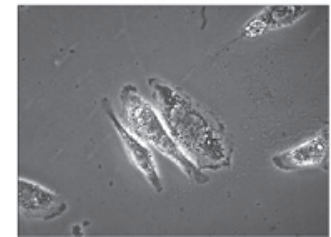

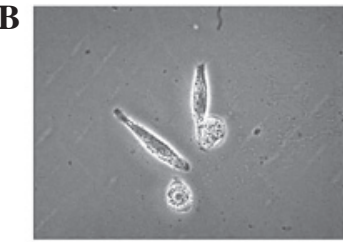

G

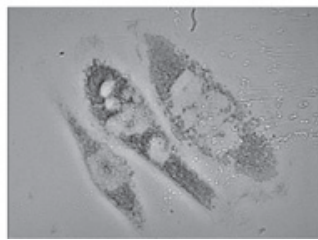

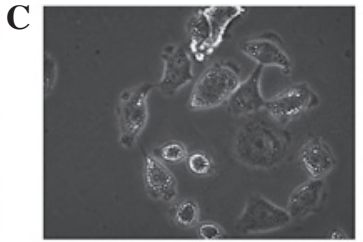

H

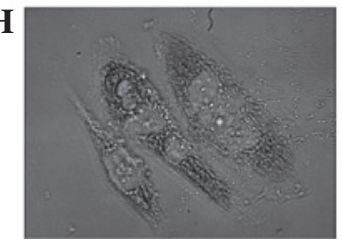

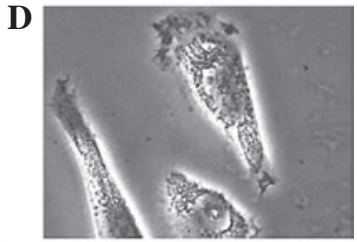

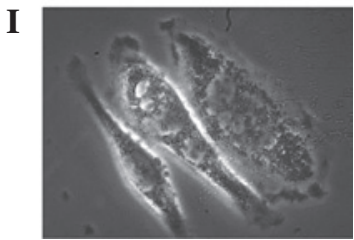

Figure 4. (A and B) Following drug screening Hep3B cells exhibited different cell morphology compared with (C-H) Hep3B-122 cells. (C and D) Cells expanded and the edges became smooth and bright with the increase in the cell size. (E-H) Vacuoles appeared in the transfection phase and gradually integrated into larger vacuoles in the center, similar to a lipid granule, $(\mathrm{C}-\mathrm{H})$ transparent droplets gathered at the edge or two poles of the cells. Magnification: (A and B), x40; (C-E), x100; (F-H), x400.
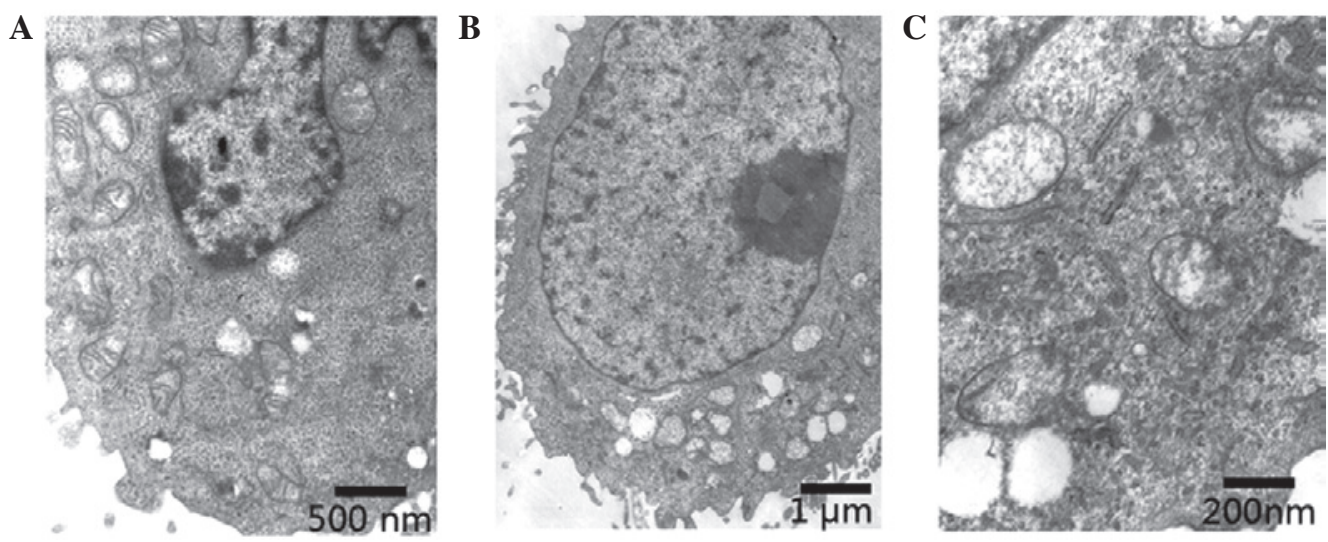

Figure 5. Transmission electron microscopy scanning. (A) Hep3B; (B and C) Hep3B-122. The nuclei of Hep3B-122 cells became bigger and were located at the edge of the nuclear envelope; the amount of heterochromatin decreased compared with Hep3B cells. (B) Hep3B-122 celss were enlarged and the cytoplasm was vacuolated and organelles were damaged; the microvilli on the cell membrane were also damaged. (C) Along with the swelling of mitochondria, the double membrane structure was not clear due to mitochondiral cavitation and the amount of heterochromatin decreased.

\section{Discussion}

Biomolecules are generated by healthy livers for export to consumer tissues of the body, including proteins, fats, carbohydrates, vitamins and minerals. Protein and fat are decomposed into amino acids and fatty acids, respectively, in the liver which contains a large number of mitochondria. Mitochondria are known as the 'energy factory' as they are the primary producer of adenosine triphosphate in cells. The balance of liver energy and material metabolism is destroyed in hepatocarcinoma and hepatocytes metabolize glucose into lactate, even in the presence of sufficient oxygen to support mitochondrial oxidative phosphorylation, a phenomenon referred as the 'Warburg Effect' (6). The liver-specific miR-122 is frequently suppressed in primary HCCs. Thus, it may be concluded that miR-122 is required to maintain and promote the aforementioned functions and metabolism. In the current study, a number of cavities of varying sizes appeared throughout the cytoplasm $60 \mathrm{~h}$ after transfection. Following the integration of the recombinant plasmid, there was high expression of miR-122. miR-122 maintains and promotes the hepatocyte's functions and metabolism. Notably, the cavitating structure was observed to be endoplasmic reticulum (ER); miRNA mediates ER more sensitively and earlier than the thousands of genes in the synthetic process of ER. (7). Cavities located closer to the nucleus were larger. A thick ER was observed with ER-associated ribosomes connected to the nucleus by nuclear pore complexes that contain central transporter linked nucleoplasmic rings with a cytoplasmic ring. Bcl-2 resides in the nuclear envelope, ER and outer mitochondrial membrane in a nonuniform distribution and is suggested to have a role in protein complexes that may be involved in certain aspects of transport (8). miR-122 targets the Bcl family directly, which is repressed by elevated levels of miR-122, and is an endogenous apoptosis regulator in these HCC-derived cell lines (9). It may be inferred that in Hep3B-122 cells, the ER may cause changes in structural absorbance.

The morphology of a cancer cell may be modulated by miRNA activity $(10,11)$ and a number of miRNA-overexpressing cells exhibited markedly different morphologies (12). miR-122 is significantly downregulated in liver cancers and restoration of miR-122 significantly decreased in vitro migration, invasion and anchorage-independent growth, as well as in vivo tumorigenesis, angiogenesis and intrahepatic metastasis in an orthotopic liver cancer model (13). The results of the present study showed that cell morphology and cell viability 
were altered. Following drug screening, Hep3B-122-treated cells appeared more translucent compared with the control group, with the appearance of a lipid granule-like vacuoles. miR-122 has previously been linked to the regulation of cholesterol and lipid metabolism (14) and the knockdown of miR-122 expression has previously been recognized to result in the downregulation of cholesterol and lipid-metabolizing enzymes (15). Conversely, in the present study the overexpression of miR-122 in Hep3B-122 cells resulted in the accumulation of lipid granule-like transparent droplets at the cell periphery or the two poles of the cells suggesting that miR-122 upregulates cholesterol and lipid metabolism. miR-122 is essential for hepatitis C virus (HCV) RNA accumulation in cultured liver cells and it stimulates the translation of HCV RNA. Therefore, virus infection and lipodystrophy separately promote the elevated expression of miR-122 in the liver although how they interact is unclear.

Although miR-122 regulates mitochondrial metabolism and its loss may be detrimental to sustaining critical liver function in a number of studies $(5,16)$, high expression of miR-122 also stimulates the translation of HCV RNA in the liver. The HCV core protein alters mitochondrial function and results directly in an increase in the cellular abundance of reactive oxygen species, with consequent increases in cellular lipid peroxidation (16). Retrospective analysis provided evidence that silencing of miR-122 downregulates the replication of $\mathrm{HCV}$ for therapeutic purpose. By contrast, miR-122, as a tumor suppressor, targets anti-apoptotic genes to inhibit the tumorigenic properties of HCC (10). Thus, miR-122 performs specific functions at various expression levels. The present study; however, did not provide an explanation for the regulation of mitochondrial metabolism. Transmission electron microscopy images provide novel evidence that elevated expression of miR-122 may not be beneficial to mitochondria. miR-122 caused an increase in the size of the nucleus and relocated the nucleus to the edge of the nucleus and in addition to the swelling of mitochondria, the double membrane structure was not as clear due to mitochondrial cavities.

In conclusion, miR-122 was observed to markedly alter the cell morphology and negatively regulate mitochondria. miR-122 function was observed to correlate with its expression level in HCC cells.

\section{Acknowledgements}

This study was financially supported by grants from the National Natural Science Foundation of China (grant no.
81160529) and the Jilin Province Science and Technology Development Project (grant no. 200905207).

\section{References}

1. Farazi TA, Spitzer JI, Morozov P and Tuschl T: miRNAs in human cancer. J Pathol 223: 102-115, 2011.

2. Chang J, Guo JT, Jiang D, et al: Liver-specific microRNA miR-122 enhances the replication of hepatitis $\mathrm{C}$ virus in nonhepatic cells. J Virol 82: 8215-8223, 2008.

3. Lagos-Quintana M, Rauhut R, Yalcin A, et al: Identification of tissue-specific microRNAs from mouse. Curr Biol 12: 735-739, 2002.

4. Chang JH, Nicolas E, Marks D, et al: miR-122, a mammalian liver-specific microRNA, is processed from hor mRNA and may downregulate the high affinity cationic amino acid transporter CAT-1. RNA Biol 1: 106-113, 2004.

5. Lanford RE, Hildebrandt-Eriksen ES, Petri A, et al: Therapeutic silencing of microRNA-122 in primates with chronic hepatitis C virus infection. Science 327: 198-201, 2010.

6. Skárka L and Ostádal B: Mitochondrial membrane potential in cardiac myocytes. Physiol Res 51: 425-434, 2002.

7. Mato JM and Lu SC: The hepatocarcinogenic effect of methionine and choline deficient diets: an adaptation to the Warburg effect? Alcohol Clin Exp Res 35: 811-814, 2011.

8. Selbach M, Schwanhäusser B, Thierfelder N, Fang Z, Khanin R and Rajewsky N: Widespread changes in protein synthesis induced by microRNAs. Nature 455: 58-63, 2008.

9. Krajewski S, Tanaka S, Takayama S, Schibler MJ, Fenton W and Reed JC: Investigation of the subcellular distribution of the bcl-2 oncoprotein: residence in the nuclear envelope, endoplasmic reticulum, and outer mitochondrial membranes. Cancer Res 53: 4701-4714, 1993

10. Lin CJ, Gong HY, Tseng HC, Wang WL and Wu JL: miR-122 targets an anti-apoptotic gene, Bcl-w, in human hepatocellular carcinoma cell lines. Biochem Biophys Res Commun 375: 315-320, 2008

11. Tavazoie SF, Alarcón C, Oskarsson T, et al: Endogenous human microRNAs that suppress breast cancer metastasis. Nature 451: $147-152,2008$

12. Sossey-Alaoui K, Bialkowska K and Plow EF: The miR200 family of microRNAs regulates WAVE3-dependent cancer cell invasion. J Biol Chem 284: 33019-33029, 2009.

13. Elson-Schwab I, Lorentzen A and Marshall CJ: MicroRNA-200 family members differentially regulate morphological plasticity and mode of melanoma cell invasion. PLoS One 5: e13176, 2010.

14. Tsai WC, Hsu PW, Lai TC, et al: MicroRNA-122, a tumor suppressor microRNA that regulates intrahepatic metastasis of hepatocellular carcinoma. Hepatology 49: 1571-1582, 2009.

15. Krützfeldt J, Rajewsky N, Braich R, Rajeev KG, Tuschl T, Manoharan M and Stoffel M: Silencing of microRNAs in vivo with 'antagomirs'. Nature 438: 685-689, 2005.

16. Burchard J, Zhang CS, Liu AM, et al: microRNA-122 as a regulator of mitochondrial metabolic gene network in hepatocellular carcinoma. Mol Syst Biol 6: 402, 2010. 